\title{
Assessment of Association between Schizophrenia and Chlamydiaceae Using Hill Criteria
}

\author{
Bekir Kocazeybek* and Fatma Kalayci \\ Department of Microbiology and Clinical Microbiology, Cerrahpasa Medical Faculty, Istanbul University, Istanbul, Turkey
}

\begin{abstract}
Schizophrenia is a complex chronic neuropsychiatric disorder of the central nervous system with worldwide prevalence. The disease is thought to have multiple etiologies, including infections, genetic predisposition, and other environmental factors. There is increasing evidence pointing to a close association between chlamydial infections and schizophrenia. Here, we review the data available in the literature on the detection of bacterial species in the family Chlamydiaceae in schizophrenia and critically analyze the association and causal relationship between Chlamydiaceae and schizophrenia following the established criteria of Hill. This analysis demonstrates a probable causal relationship between Chlamydiaceae and schizophrenia. The identification of microbial agents associated with the etiopathogenesis of schizophrenia may provide new insight into the diagnosis, treatment and prevention of the disorder.
\end{abstract}

Keywords: Schizophrenia; Chlamydiaceae; Hill criteria

\section{Introduction}

The acknowledgement that pathogenic microorganisms can cause chronic diseases has provided a new perspective on infectious diseases. As Judith Miklossy mentioned that Barry Marshall and Robin Warren's discovery that Helicobacter pylori causes stomach ulcers [1] together with the ensuing report that the bacterial agent Tropheryma whippeli is associated with Whipple's disease [2] were remarkable advances, leading to novel approaches to the diagnosis, prognosis and treatment of these diseases [3]. More recently, infectious agents have also been reported to cause atherosclerosis [4], cardio [5] and cerebrovascular diseases [6], diabetes mellitus [7], chronic pulmonary [8] and inflammatory bowel diseases [9], neurological [10] and neuropsychiatric diseases [11,12].

Many infections were reported to induce symptoms of mental illness, but these symptoms may disappear after recovery but some symptoms may not disappear if an acute infection becomes chronic. Schizophrenia is believed as one of these mental illnesses and also is a multifactorial neuropsychiatric disease that is characterized as a disorders cluster of unknown etiology. Family, twin and adoption studies have shown that this disease is highly hereditary and identified genetic determinants responsible for its transmission [13]. In addition to these genetic determinants, birth season [14], urban birth [15], household crowding [16], migration [17], obstetrical complications [18], and close cat contact frequency [11] have been considered as epidemiologic parameters in the schizophrenia etiology. Many epidemiologic researches concentrate on microorganism exposure arising from environmental factors [19-24] and perinatal/postnatal infections [25]. The most investigated microorganisms are brain development affecting agents in human or animal models. In studies addressing perinatal exposure those agents most frequent suggested as causing schizophrenia (odds ratio) are rubella [26], Toxoplasma gondii [25], influenza A [27], Cytomegalovirus (CMV) [28], Chlamydia trachomatis [29], Human papillomavirus type 16 (HPV 16) [29] and Herpes simplex virus-2 (HSV-2) [21]. Similarly, much emphasis has been given to the potential contribution of viral meningitis (during the neonatal and adolescent periods) to schizophrenia risk [30]. In a literature research, we have identified that the agents most correlated with schizophrenia and permanent psychosis, especially on the adult population, are CMV [31], Herpes simplex virus-1 (HSV-1) [32], Borna Disease virus (BDV) [23], Human Endogen Retrovirus-W (HERV-W) [33], Human immunodeficiency virus 1 (HIV-1) [34],
Borrelia burgdorferi [35], Treponema palladium [19], and Chlamydia trachomatis, Chlamydophila psittaci and Chlamydophila pneumoniae from Chlamydiaceae members [24]. The principal reasons for focusing on these infectious agents is because it has been long thought that these agents have the ability to settle persistently in the central nervous system, together with the observation of neurological and psychiatric symptoms in some genetically predisposed people [36]. However, the existence of limitations in case-control studies on adults has cast doubt on the validity of some schizophrenia studies. As examples, such studies are often accompanied with discussions on: infectious agent antibody exposure across a series of demographic parameters (e.g., age, gender, race, socio-economic status and geographical location) $[37,38]$ antibody titres determination only in the period immediately after the beginning of symptoms [39] and how infectious agent exposure can increase hospitalization period and/or homelessness; and changes in the host immune response caused by several applied treatments [40].

Chlamydiaceae is a family that contains Chlamydia and Chlamydophila species and has been implicated in schizophrenia's etiopathogenesis by a limited number of studies. Chlamydiaceae members may show localization on tissues in a persistent manner as they follow biphasic life cycles and are obligated intracellular bacteria. While the C. trachomatis causes the main oculogenital diseases and trachoma $[41,42]$, C. pneumoniae had been investigated in the context of several manifestations, including bronchitis, atypical pneumonia [41], coronary artery disease [4], arthritis [43], encephalitis, meningitis [44], neurodegenerative diseases (Alzheimer's disease, multiple sclerosis) [45,46] and neuropsychiatric disorders (schizophrenia, autism, bipolar disorder) [24]. C. psittaci has been associated with pneumonia [47], encephalitis [48], stroke, confusion, and hearing

*Corresponding author: Bekir Kocazeybek, Department of Microbiology and Clinical Microbiology, Cerrahpasa Medical Faculty, Basic Science Building, Floor 2 Fatih, İstanbul, Turkey, Tel: 00-90-532-6168150; Fax:00-90-212-6322122; E-mail: bzeybek@istanbul.edu.tr

Received January 10, 2015; Accepted February 17, 2015; Published February 24, 2015

Citation: Kocazeybek B, Kalayci F (2015) Assessment of Association between Schizophrenia and Chlamydiaceae Using Hill Criteria. J Psychiatry 18: 250 doi: 10.4172/2378-5756.1000250

Copyright: (C) 2015 Kocazeybek B, et al. This is an open-access article distributed under the terms of the Creative Commons Attribution License, which permits unrestricted use, distribution, and reproduction in any medium, provided the original author and source are credited 
loss [49]. The reason of the association of Chlamydiaceae with many different clinic manifestations and especially with schizophrenia, is its ability to interact with its host by remaining on body tissues, causing chronic infection and relapse features. Some data has suggested that Chlamydiaceae could cross the blood-brain barrier. One piece of supporting evidence for this is the recurrence of psychosis upon increasing interleukin-2 ( IL-2) levels in patients [50]. IL-2 is synthesized as autocrine by T helper 1 (Th1) cells, resulting in Th1 cell proliferation and induces cytokine production from $C$. pneumoniae infected monocytes/macrophages [51]. It has been thought that with that process it might be resulted in favour of proliferous immune system cells' different neurotrophin (NT) synthesis [27]. NT molecules play an important role in the development, function, and survival of neuronal cells [52]. Moreover, these molecules are neuromodulators of monoamiergic and cholinergic systems, which regulate synaptic activity and neurotransmitter synthesis. Brain Derived Neurotrophic Factor (BDNF) and Neurotrophin-3 (NT-3) are known to modulate dopamine, gamma-aminobutyric acid (GABA) ergic, and serotonergic receptors $[53,54]$.

Neurotrophins are synthesized from microglia $[55,56]$ and Th1 and Th2 cells [57,58], and their effects upon target neurons and tissues of the peripheral and central nervous systems have been demonstrated $[55,56]$. Relevant to this, it was asserted that in cultured microglial cells, lipopolysaccharide (LPS)-induction increases the expression of Nerve Growth Factor (NGF), BDNF and Neurotrophin 4/5 (NT 4/5) [58]. In addition, it has reported that Th 1 and Th 2 could produce NT-3 and BDNF respectively, and that increases in the synthesis of these neurotrophins could be the result of a Th1- or Th2-based immune response $[57,58]$. A few studies have reported about association between Chlamydiaceae infections and the risk of schizophrenia. The aim of this review is to assessment of association between schizophrenia and Chlamydiaceae infections using hill criteria and results of other studies. Therefore, we aimed to make an assessment of the role of the Chlamydiaceae family using data from several recent case-control studies on schizophrenia etiopathogenesis. Our approach is based upon the Sir Austin Bradford Hill criteria (strength, consistency, specificity, temporality, biological gradient, plausibility, coherence, experimental evidence, analogy) for interpreting agent exposure and clinic association.

\section{Analysis of Causal Relationship Between Chlamydiaceae and Schizophrenia following Hill's Criteria}

\section{Strength}

In a limited number of studies related to the probable relationship between schizophrenia and Chlamydiaceae, and performed using both serologic and molecular techniques, it has been reported that the frequency of a chronic infection due to C. pneumoniae in schizophrenic patients shows a statistically significant difference against that of the control group [24,59]. In Kalayci's [59] study, 50 schizophrenic and 35 healthy control group were included and $28 \%$ of the 50 schizophrenic patients and $2.85 \%$ of the 35 controls had chronic C.pneumoniae seropositivity. The calculated OR value (13.2), evaluated as high by univariant calculation, and the calculated independent risk factor (39.8) determined multivariant analyse, were in line with other studies. Fellerhoff et al. [24] analyzed frequencies of three types of Chlamydiaceae in schizophrenic patients $(n=72)$, random controls $(n=225)$ and hospital-patient controls $(n=36)$, they found chlamydial infection in $40.3 \%$ of the schizophrenic patients compared to $6.7 \%$ in the controls. They determined an OR of 24.39 for the C. psittacischizophrenia association when using molecular based studies. The same researchers have found 4-fold greater Chlamydophila DNA in the frontal cortex of schizophrenia patients' post-mortem brain tissues than in controls and reported a significant difference between the two groups in terms of Chlamydophila infection frequency $(\mathrm{p}<0.05)$ [60]. For C. trachomatis, no significant difference in bacteria DNA detection was found between the control groups and schizophrenic patients [24]. In a Korean study, C. pneumoniae and C. trachomatis in 96 schizophrenia and 50 control subjects was investigated using enzyme-linked immunosorbent assay. They found C. pneumoniae IgG seropositivity in $64(66.7 \%)$ patients and $35(70.0 \%)$ controls and also found C. trachomatis IgG seropositivity in 27 (38.6\%) patients and 9 (18\%) controls. An odds ratio of 2.86 has been reported for the $C$. trachomatis-schizophrenia association and a significant difference between the two groups has been reported for past infection rate $(p<0.05)$ [61]. In another study, 31 patients with schizophrenia and 30 controls were investigated and $25.8 \%$ and $0 \%$ C. trachomatis IgG seropositivity were found in patients with schizophrenia and controls, respectively. A significant difference $(\mathrm{p}<0.05)$ was reported for past $C$. trachomatis infection rate in schizophrenic patients compared to healthy controls [62]. The studies of Chlamydiaceae antibodies and DNAs as risk factors in adults with schizophrenia have reported odds ratios in the range of 2.86-24.9. This would be considered a high degree of association in most studies but serological assays have limitations like crossreactivity among Chlamydiaceae species and with other organisms. In the study of Fellerhoff et al. [24], the association of schizophrenia with Chlamydiaceae infections was highly significant $(\mathrm{OR}=9.43)$, especially with C.psittaci $(\mathrm{OR}=24.39)$ but $C$. psittaci prevalence is not high in the general population, but is typically more common in bird handlers [63] This is the limitation for the C. psittaci-schizophrenia association. In the study of Fellerhoff et al. [60], both C. psittaci and C. pneumoniae DNA were detectable in peripheral blood and post-mortem brain tissue samples, as are antibody titers of $C$. trachomatis. Their results appear to satisfy Hill's "Strength" criteria for schizophrenia association but needs more comprehensive molecular studies. The high odds ratios of the studies seem to satisfy the strength criteria of this association.

\section{Consistency}

To satisfy Hill's "consistency" criteria, the Chlamydiaceaeschizophrenia association should be reproducible across different places, times, situations and populations [64]. Despite the limited number of available studies concerning the ethiopathogenesis of schizophrenia and its association with Chlamydiaceae, especially the increased frequency of C. pneumoniae and C. psittaci, DNA determined in peripheral blood mononuclear cells (PBMC) [24] and post-mortem brain tissue [60] are remarkable findings supporting this relation. Also serological findings support this relation. Kalayci's findings [59], together with others [24,60-62], support the possibility that a relationship exists between schizophrenia and Chlamydiaceae infections.

The consistency criteria is supported by identified associations in studies with patients from Germany [24,62], Korea [61], America [60] and Turkey [59], which were conducted at different times. It is important to note that antibody titers prove only that encounter with the infectious agent has occurred, but these titers do not prove that the infectious agent is still present in the body. Only a PCR assay with specific DNA probes can verify a persistent infection with a microbe. This is the limitation of the study and there are very few studies $[24,60]$ detecting C. pneumoniae and C. psittaci DNA .

\section{Specificity}

Given that schizophrenia does not occur in all cases of 
Chlamydiaceae infection, nor does every schizophrenic case have past Chlamydiaceae infection, it has been suggested that psychosis is only triggered only genetically predisposed people, as in T. gondii infections [39]. For example, it had been claimed that $C$. psittaci increases the risk factor for schizophrenia by 50 -fold in those carrying the HLA-A10 genotype. It has also been reported that HLA-A10 genotype carrying schizophrenics are widely infected with Chlamydophila [24]. In order to support the specificity criteria, it is important to demonstrate that the patients had been infected by the agent before psychosis developed. Despite the increased risks, the majority of individuals who have antibodies to Chlamydiaceae do not have schizophrenia or other psychiatric diseases. Thus, the effect of Chlamydiaceae infection is not specific in terms of schizophrenia. It will be important to further define specificity in terms of genetic susceptibility, timing of infection, and other factors which might explain individual differences in response to infectious agents [39]. In addition, it would be important to determine whether different infection agents' (especially neurotrophic agents) have similar effects in psychosis development via triggering similar inflammatory responses. The co-infections hypothesis should also be considered.

\section{Temporality}

In order to satisfy the temporality criteria, it will be necessary to demonstrate that infection with Chlamydiaceae preceded schizophrenia in an epidemiological study. Thus, the agent exposure must be shown before the prenatal or early life stages (i.e., before disease manifestation). In addition, the agent's DNA load efficiency in the infected persons could be important for triggering psychosis through induction of inflammation and immune response. Therefore, determining DNA existence and copy count will be essential in interpreting the in vivo effect. Before, evaluation the association in terms of temporality, we need to consider the neurodevelopment theory of schizophrenia in greater depth. The neurodevelopment theory of schizophrenia suggests abnormalities in early neurodevelopment as a possible cause of disorder $[65,66]$. According to the neurodevelopmental theory, the etiology of schizophrenia may involve pathologic processes, caused by both genetic and environmental factors, that begin before the brain approaches its adult anatomical state in adolescence [67]. HSV-2 and T. gondii were reported up to a twofold increased risk of schizophrenic psychosis in some studies. Exposure to influenza, and other infections during early stages of gestation may mediate risk of psychotic illness. Conversly, no evidence was reported between prenatal HSV-1 or CMV infection and adult psychotic disorders. In common sense, the suggestion is inflammatory cytokines may mediate risk of psychotic illness associated with prenatal infection. The neurodevelopment theory of schizophrenia summarize, briefly maternal infection or increased inflammatory cytokines during pregnancy may be associated with both structural and functional brain phenotypes relevant to schizophrenia in the offspring [68]. Khandaker et al. [68] made a remarkable classification about the effect of prenatal infections for foetal development. Mechanisms by which prenatal infections interfere with foetal development and contribute to risk of adult schizophrenia may include (i) direct interference with neurodevelopment by infectious agents or antibodies; maternal infection during pregnancy with neurotrophic agents, such as T. gondii, HSV and rubella, can interfere directly with foetal neurodevelopment [69]. (ii) induction of autoimmunity; it has been proposed that certain prenatal infections such as influenza may increase the risk of adult psychotic illness through induction of autoimmunity [70] (iii) the involvement of mediators of acute infection; developmental effects of acute infections such as influenza may be mediated by hyperthermia and foetal hypoxia $[71,72]$ (iv) reprogramming of the hypothalamic-pituitary-adrenal(HPA) axis; infection can increase foetal exposure to maternal glucocorticoids by inhibiting the placental enzyme 11-beta-hydroxysteroid dehydrogenase type 2 [73]. Excess glucocorticoids can reprogramed the HPA axis in the off spring, lead into either increased basal secretion or enhanced stress related secretion of glucocorticoids later in life [74]. Thus, increased basal cortisol levels observed in some cases of schizophrenia [75] may be related to maternal infections during pregnancy (v) activation of the innate immune system and effects of inflammatory cytokines; as increased risk of psychosis has been observed for a diverse range of prenatal infections, it has been proposed that they may share a common pathway to exert pathogenic effects on the foetal brain. One such mechanism involves activation of the innate immune system and release of proinflammatory cytokines [76] (vi) interaction between host and microbe genome and/or environmental factors; variations in the gene encoding IL-10 are reported to be associated with susceptibility to both CMV infection and schizophrenia [77,78]. All of the above mechanisms by which prenatal infections with Chlamydiaceae may interfere with foetal development and contribute to risk of adult schizophrenia but needs comprehensive prospective cohort studies to clarify the role of Chlamydiaceae in the etiopathogenesis of schizophrenia. However, to confirm these criteria will require studies in which children are followed prospectively. The frequency of $C$. pneumoniae titers increase with age. The high titers in the elderly are probably "boost" resulting from frequent re-infections. The seropositivity of $C$. pneumoniae in $<5$ years old increases to approximately $40 \%$ at 10 years of age. Seropositivity is greater than $75 \%$ in the elderly [79]. In an epidemiological study in Finland it was found similar infection rates for children: $55 \%$ for children 10-14 years of age and 70\% for children 15-19 years of age [80]. As Fellerhoff [60] mentioned that the onset of schizophrenia typically occurs between the late teens and the mid-30s; onset prior to adolescence is rare. These statistical studies suggest that $C$. pneumoniae infects individuals before the onset of schizophrenia [60]. Counterfactual argument in the application of temporality is somewhat controversial since most studies analyze antibody levels in individuals with the symptoms of schizophrenia and it is possible that these individuals are exposed to Chlamydiaceae or other infectious agents after the onset of their symptoms. Serological assays are not adequate due to several limitations; for example, there is high cross-reactivity among Chlamydiaceae species themselves and with other organisms [24] and it is difficult to distinguish between a chronic infection and a former exposure to the pathogen(s). For this reason, there is a need for prospective cohort studies that assess exposure in adults prior to the onset of symptoms [39].

\section{Biological gradient}

This criterion is related with increased exposure to an environmental agent should lead to an increased rate of disease. In addition, determining the Th1 and Th2 immune responses, which have important roles in neuronal plasticity, immune system activation against Chlamydiaceae members, and change in neurotrophin levels (e.g., BDNF and NT-3, which are thought to be released from monocytes and microglia in case of infection), will be important for investigating the association. Moreover, psychosis recurrence rate in patients with high immunological markers levels (e.g., IL-2, IL6 , and TNF-alfa), indicative of infection, as well as quantification of those markers' levels provides further supportive information for a schizophrenia-infection association. As a result of changes in those parameters mentioned above, we are of the opinion that psychosis development in people with affected nervous system is quantitatively linked to the infection level [81-83]. Moreover, high (although not 
statistically significant) levels of neurotrophin-3 in C. pneumoniae seropositivity cases with schizophrenia relative to seronegative cases have been determined in our previous study [59]. The nervous system might be affected by changes in the immune system [24]. Because of Chlamydiaceae studies have been based mostly on serological methods, to assess the quantitative measures of exposure is difficult for example by molecular methods. For above reasons, biological gradients cannot be accurately measured. Improved laboratory methods like molecular may detect quantitative markers of exposure [39]. In order to support the causality in association of Chlamydiaceae infections and the risk of schizophrenia, sensitive PCR techniques were needed.

\section{Plausibility}

While referring to this criterion, Sir Hill cites an advice of the Sherlock Holmes character, "...when you have eliminated the impossible, whatever remains, however improbable, must be the truth". As Yolken and Torrey mentioned, this factor is open to subjective evaluation [38]. As a result there are many strong proponents of such theories related to these diseases [38]. However, in the case of infection with a microorganism, persistent settlement on brain indication [60] and when the results were also considered, researching Chlamydiaceae's, one of the infectious agents, roles in schizophrenia aetiopathogenesis may be stay in "plausibility" borders C. psittaci has been reported also in a series of neurologic disorders, like meningitis [84], cranial nerve palsy including sensory hearing loss [85], encephalitis, confusion [86] seizures, and other transient focal neurologic signs [87]. Not all individuals suffer from the same disease symptoms and to the same extent after being infected with Chlamydiaceae species. There are some limitations for this criteria because Chlamydophila spp. were not present in all patients with schizophrenia. However, other microbial infections may also be the main pathogenic factor in the development of schizophrenia. Even this limitations, using PCR and sequence analysis, Fellerhoff et al. [60] found Chlamydophila DNA to be four times greater in patients with schizophrenia than in controls (schizophrenia: $\mathrm{N}=34, \mathrm{OR}=5.08$ ) [60]. Previously, they identified C. psittaci and/or $C$. pneumoniae DNA by PCR in blood samples of $40 \%$ of patients with schizophrenia from Germany, compared to $6 \%$ of control individuals [24]. They hypothesized that Chlamydophila (or other Chlamydiaceae) may persist not only in monocytes but also in the brain cells of patients with schizophrenia. Whereas this criteria, which is supported by genetic susceptibility, can be evaluated according to the immunogenetic predisposition of the patient. Coherence, experimental, and analogenic evidences support the hypothesis that exposure to persistent or chronic Chlamydiaceae infections can trigger psychosis. Unfortunately, the scarce of studies doesn't support the causality in the association of Chlamydophila and schizophrenia for the present.

\section{Coherence}

As proposed by Hill, the cause-and-effect interpretation of the data should not seriously conflict with the generally known facts of the natural history and biology of the disease [64]. Rather, the examined "coherence" concept in a disease epidemiology should be supported by the "plausibility" concept, and vice versa $[39,64]$. Chlamydiaceae, which is a known intracellular pathogen, can be evaluated as a major agent due to having neurotropism, especially in neurologic and psychiatric patients [24]. Strong evidences with reference to prenatal and maternal infections and schizophrenia risk increase are common in the literature. The effects of infections during pregnancy, especially with those pathogens belonging to the TORCH group (e.g., T. gondii, Rubella, Cytomegalovirus, Herpes simplex virus) and those related to influenza and inflammatory cytokines had been reported [27,29]. These infections are been thought to cause mental retardation, cerebral hypoplasia, and other neonatal brain and behaviour abnormalities. The same prenatal infections are also been thought to cause malfunction related to brain structure and function [29]. In this sense, we consider maternal or prenatal infections of $C$. trachomatis, which is a member of Chlamydiaceae family, and the chlamydial non-gonococcic urethritis factor, as non-excludable from schizophrenia aetiopathogenesis [88]. It is well known that $C$. trachomatis is associated with manifestations such as conjunctivitis [89], nasopharyngitis [90], pneumonia [91] and rarely myocarditis [92], encephalitis [93]. An American study conducted in 2001 assessed the contribution of maternal infections during pregnancy to schizophrenia (or the other psychoses) development in adulthood and reported that neonatal HSV-2 seropositivity increased risk. In the same study, no significant differences were found between the case and control mothers of C. trachomatis antibody [29]. Whereas an animal study has shown that $C$. trachomatis might have been infected astrocytes in vitro in the same way as toxoplasma, and might had been replicated to rat brain astrocytes [94]. These findings suggest that future acute encephalitis can result from a maternal or prenatal C. trachomatis infection.

In addition, according to the ability of Chlamydiaceae to pass the blood-brain barrier via infecting monocytes, as well as its ability to infect glial, microglial, and neural cells, it was suggested that persistent Chlamydiaceae infection might trigger the process leading to schizophrenia, destroying the balance present between the immune and nervous systems. It is emphasized that, in cases with chronic Chlamydiaceae infection, high level inflammation markers and neurotrophins (e.g., BDNF and NT-3) can be evaluated as an indicator of a schizophrenia-Chlamydiaceae relationship. In the case of Chlamydiaceae infection, the ability of $C$. psittaci to infect the human brain and establish persistent infection in early life is partly consistent with the finding of neurodevelopmental abnormalities in individuals with schizophrenia. There are a few studies and they are related with C. psittaci. All these observations indicate that, the association is not in harmony with the natural history and biology of schizophreniaChlamydiaceae except C. psittaci. There is also need comprehensive studies to prove the causality using the coherence criteria.

\section{Experimental evidence}

A possible Schizophrenia-Chlamydiaceae association is supported by a few studies that have been conducted at various periods and within various countries, as we mentioned above $[24,60,61]$. In addition to this we believe that this criterion is best supported with positive results of adaptive immune treatment studies, such as the studies by Wank [95] and Fellerhoff et al. [96], in which targeted regression of symptoms was achieved by with immune paracellular balance of BDNF and NT-3 release, while providing immune cell subpopulation together with azithromycin treatment to schizophrenic patients that tested positive for Chlamydiaceae. Experimental evidence supports the possible link between the suppression of Chlamydiaceae replication in vitro and the treatment of human psychiatric diseases. For example, treatment with in vitro-activated immune cells together with azithromycin showed mental improvements in patients that did not depend on treatment with antipsychotic drugs but patients reported benefits only for short periods, i.e. for days or a week [96]. These findings are also supported by others [97]. Further research and clinical trials are needed to prove these criteria.

\section{Analogy}


This criteria describes briefly as "different agents can invoke similar pathogenic mechanisms". As we mentioned above, schizophrenic psychosis has been proposed to arise as a result of infection with other neurotropic organisms, such as Rubella virus, influenza virus, and HSV-2, CMV, T. gondii have been documented to lead to increased risks in different study populations and they are especially intracellular pathogens showing neurotrophic features and triggering similar immunological pathways [39]. These findings suggest that, in the case of human psychiatric diseases, infections caused by neurotropic organisms are likely to display analogy.

\section{Conclusions}

Schizophrenia is a complex disease which has environmental and genetic factors in its etiopathogenesis. In the light of restricted and few studies related with the association between Chlamydiaceae and schizophrenia, and within the framework of Hill's criteria, the majority of the evidence may appear to support a probable association between Chlamydiaceae and schizophrenia. The association between Chlamydiaceae infections and schizophrenia may be supported by factors such as strength, consistency, experimental evidence, and analogy. Although, the main factor which is not satisfied is specificity, at the same time the obtained data remains insufficient for satisfying the specificity criterion since Chlamydiaceae is probably one of several infectious agents that can lead to schizophrenia in genetically susceptible individuals. In addition, because of there is no prospective cohort study in the literature, the temporality criterion cannot be satisfied without demonstrating that the agent is encountered before schizophrenia diagnosis, which would likely require a cohort prospective study design. Also, there are no enough evidences with a few studies to prove causality in this relation for biological gradient, cohorence and plausibility. Genetic compatibility is an additional criteria proposed by Yolken and Torrey [39]. This criterion requires that the clinical expression of an environmental factor associated with a disease be consistent with genetic factors that are known to operant in the target disease. Chlamydiaceae infections, especially C. psittaci, fulfill this criteria. This additional criteria supports the causality in this association by Fellerhoff et al. [24]'s findings. They found that carriers with HLA-A10 and infection by C. psittaci showed the highest risk of schizophrenia $(\mathrm{OR}=50.0)$.

Finally, the ultimate proof of the association will require the demonstration that the prevention or treatment of Chlamydiaceae infections leads to a decrease in the rate of schizophrenia. Experimental azitromycin combined adaptive cell treatment findings can be considered as important evidence for this association $[95,96]$. Remission in treated patients' symptoms will provide further insight into the association of Chlamydiaceae with schizophrenia and help to develop new diagnosis and treatment methods against schizophrenia, a condition associated with devastating economic, social and mental health consequences. For now, although a Chlamydiaceaeschizophrenia association (as determined using Hill's criteria) appears likely, the evidence remains less convincing than that for a Toxoplasmaschizophrenia association. To address this, long-term experimental studies using serial epidemiological assessment and specimen blood samples, together with serologic- and molecular-based studies directed to detect infection agents from post-mortem tissues, will be beneficial to determining the role of Chlamydiaceae and other infection agents in schizophrenia.

\section{Limitations}

In literature survey, we couldn't find a large number of studies which support the association between Chlamydiaceae and schizophrenia than the other studies with evidences supporting the role of toxoplasmosis in the pathogenesis of schizophrenia. Therefore, the existence of a few studies related with Chlamydiaceae-schizophrenia association can be accepted as a major limitation of our review. The main factor which is not satisfied in the evaluated studies is specificity, since Chlamydiaceae is probably one of several infectious agents that can lead to schizophrenia in genetically susceptible individuals. There is also a need for additional studies of temporality in terms of adult exposure to Chlamydiaceae and the onset of schizophrenia. This association can best be analyzed by prospective cohort studies in which individuals are assessed prior to the onset of symptoms. In addition, most of the studies used serological assays and they are not adequate due to several limitations; for example, there is high cross-reactivity among Chlamydiaceae species themselves and with other organisms and it is difficult to distinguish between a chronic infection and a former exposure to the pathogen(s). As examples of limitations; In the study of associations between Chlamydiaceae infections, schizophrenia and risk of HLA-A10, Fellerhoff et al. [24] concluded that their findings do not prove that Chlamydiaceae are causative for the schizophrenic disorder or that they affect severity or disease duration and even the PCR technique of their study had high specificity but may have missed some chlamydial infections if the amount of Chlamydiaceae-DNA was too low ( $\leq 1$ IFU) for detection. Therefore, they reported the sensitivity limits of PCR technique as a limitation in their study. Furthermore, as a different limitation in the same study, they also stated that the heterogeneity of the schizophrenic disorder may reflect several pathogenic environmental factors as well as a variable genetic endowment. In other study of Fellerhoff et al. [60], they analyzed DNAs for Chlamydiaceae-DNA from the frontal cortex of brain collection. The existence of no available material to perform serological investigations was their study's limitation and they had no chance to compare the PCR results with antibody titers of patients. Also, the number of investigated brain samples and the sampling from microglia-poor gray matter has limited their study.

In Park et al's study [61], they reported their small sample size as the limitation of their study and they also stated that it was not possible to evaluate the rate of sexual activity in patients and controls. This aspect seems to be important, as C. trachomatis is a sexually transmitted disease. They reported this as the second limitation of their study.

\section{Conflict of Interest}

The authors declare that there are no conflicts of interest.

\section{Acknowledgment}

The authors would like to thank Dr. Hrisi Bahar Tokman, Dr. Suat Sarıbas (Cerrahpasa Medical Faculty, Turkey), for her assistance with the study and Banu Tufan MD for her reading of the manuscript.

\section{References}

1. Marshall BJ, Warren JR (1984) Unidentified curved bacilli in the stomach of patients with gastritis and peptic ulceration. Lancet 1: 1311-1315.

2. Dutly F, Altwegg M (2001) Whipple's Disease and "Tropheryma whippelii". Clin Microbiol Rev 14: 561-583.

3. Miklossy J (2011) Alzheimer's disease- a neurospirochetosis. Analysis of the evidence following Koch's and Hill's criteria. J Neuroinflammation 8: 90.

4. Saikku P (1999) Epidemiology of Chlamydia pneumoniae in atherosclerosis. Am Heart J 138: S500-503.

5. Renvert S, Pettersson T, Ohlsson O, Persson GR (2006) Bacterial profile and burden of periodontal infection in subjects with a diagnosis of acute coronary syndrome. J Periodontol 77: 1110-1119. 
Citation: Kocazeybek B, Kalayci F (2015) Assessment of Association between Schizophrenia and Chlamydiaceae Using Hill Criteria. J Psychiatry 18: 250 doi: 10.4172/2378-5756.1000250

Page 6 of 7

6. Wu T, Trevisan M, Genco RJ, Dorn JP. Falkner KL et al. (2000) Periodontal disease and risk of cerebrovascular disease: the first national health and nutrition examination survey and its follow-up study. Arch Intern Med 160: 2749-2755.

7. Marrollo M, Latella G, Melideo D, Storelli E, lannarelli R, et al. (2001) Increased prevalence of Helicobacter pylori in patients with diabetes mellitus. Dig Liver Dis 33: 21-29.

8. Kocazeybek B (2003) Chronic Chlamydophila pneumoniae infection in lung cancer, a risk factor: a case-control study. J Med Microbiol 52: 721-72.

9. Eaton SR, Mazuski JE (2013) Overview of severe Clostridium difficile infection. Crit Care Clin 29: 827-39.

10. Wozniak MA, Mee AP, Itzhaki RF (2009) Herpes simplex virus type 1 DNA is located within Alzheimer's disease amyloid plaques. J Pathol 217: 131-138.

11. Yuksel P, Alpay N, Babur C, Bayar R, Saribas S, et al. (2010) The role of latent toxoplasmosis in the aetiopathogenesis of schizophrenia--the risk factor or an indication of a contact with cat? Folia Parasitol (Praha) 57: 121-128.

12. Torrey EF, Yolken RH (2003) Toxoplasma gondii and schizophrenia. Emerg Infect Dis 9: 1375-1380.

13. Levinson DF (2005) Meta-analysis in psychiatric genetics. Curr Psychiatry Rep 7: 143-51.

14. Torrey EF, Miller J, Rawlings R, Yolken RH (1997) Seasonality of births in schizophrenia and bipolar disorder: a review of the literature. Schizophr Res 28: $1-38$.

15. Mortensen PB, Pedersen CB, Westergaard T, Wohlfahrt J, Ewald H, et al. (1999) Effects of family history and place and season of birth on the risk of schizophrenia. N Engl J Med 340: 603-68.

16. Agerbo E, Torrey EF, Mortensen PB (2001) Household crowding in early adulthood and schizophrenia are unrelated in Denmark: a nested case-control study. Schizophr Res 47: 243-66.

17. Cantor-Graae E, Selten JP (2005) Schizophrenia and migration: a metaanalysis and review. Am J Psychiatry 162: 12-24.

18. Geddes JR, Verdoux H, Takei N, Lawrie SM, Bovet P, et al. (1999) Schizophrenia and complications of pregnancy and labor: an individual patient data meta-analysis. Schizophr Bull 25: 413-23.

19. Kohler CG, Pickholtz J, Ballas C (2000) Neurosyphilis presenting as schizophrenialike psychosis. Neuropsychiatry Neuropsychol Behav Neurol 13: 297-302.

20. Prasad KMR, Shirts BH, Yolken RH, Keshavan MS, Nimgaonkar VL (2007) Brain morphological changes associated with exposure to HSV1 in firstepisode schizophrenia. Mol Psychiatry 12: 105-113.

21. Mortensen PB, Pedersen CB, Hougaard DM, Nørgaard-Petersen B, Mors O, et al. (2010) A Danish National Birth Cohort study of maternal HSV-2 antibodies as a risk factor for schizophrenia in their offspring. Schizophr Res 122: 257-263.

22. Aslan M, Kocazeybek B, Turan N, Karakose AR, Altan E, et al. (2012) Investigation of schizophrenic patients from Istanbul, Turkey for the presence of West Nile virus. Eur Arch Psychiatry Clin Neurosci 262: 173-177.

23. Karakose AR, Yuksel P, Turan N, Ziver T, Saribas, S, et al. (2011) Does borna disease virus (BDV) have a role in the etiopathogenesis of schizophrenia? Afr $\mathrm{J}$ of Microbiol Res 5: 1062-1069.

24. Fellerhoff B, Laumbacher B, Mueller N, Gu S, Wank R (2007) Associations between Chlamydophila infections, schizophrenia and risk of HLA-A10. Mo Psychiatry 12: 264-272.

25. Brown AS, Schaefer CA, Quesenberry, CP Jr, Liu L, et al. (2005) Maternal exposure to toxoplasmosis and risk of schizophrenia in adult offspring. Am J Psychiatry 162: 767-73.

26. Brown AS, Susser ES (2002) In utero infection and adult schizophrenia. Ment Retard Dev Disabil Res Rev 8: 51-7.

27. Brown AS, Begg MD, Gravenstein S, Schaefer CA, Wyatt RJ, et al. (2004) Serologic evidence of prenatal influenza in the etiology of schizophrenia. Arch Gen Psychiatry 61: 774-80.

28. Shirts BH, Prasad KM, Pogue-Geile MF, Dickerson F, Yolken RH, et al. (2008) Antibodies to cytomegalovirus and Herpes Simplex Virus 1 associated with cognitive function in schizophrenia. Schizophr Res 106: $268-74$

29. Buka SL, Tsuang MT, Torrey EF, Klebanof MA, Bernstein D, et al. (2001) Maternal infections and subsequent psychosis among offspring. Arch Gen Psychiatry 58: 1032-7.

30. Koponen $\mathrm{H}$, Rantakallio P, Veijola J, Jones $\mathrm{P}$, Jokelainen, et al. (2004) Childhood central nervous system infections and risk for schizophrenia. Eur Arch Psychiatry Clin Neurosci 254: 9-13.

31. Leweke FM, Gerth CW, Koethe D, Klosterkötter J, Ruslanova I, et al. (2004) Antibodies to infectious agents in individuals with recent onset schizophrenia. Eur Arch Psychiatry Clin Neurosci 254: 4-8.

32. Torrey EF (1986) Functional psychosis and viral encephalitis. Integr Psychiatry 4: 224-236.

33. Karlsson H, Bachmann S, Schroder J, McArthur J, Torrey EF, et al. (2001) Retroviral RNA identified in the cerebrospinal fluids and brains of individuals with schizophrenia. Proc Natl Acad Sci USA 98: 4634-4699.

34. Sewell DD (1996) Schizophrenia and HIV. Schizophr Bull 22: 465-473.

35. Fallon BA, Nields JA (1994) Lyme disease: a neuropsychiatric illness. Am J Psychiatry 151: 1571-83

36. Quinn JP, Dalziel RG, Nash AA (2000) Herpes virus latency in sensory ganglia-a comparison with endogenous neuronal gene expression. Prog Neurobiol 60: 167-179.

37. Nahmias AJ, Lee FK, Beckman-Nahmias S (1990) Sero-epidemiological and -sociological patterns of herpes simplex virus infection in the world. Scand Infect Dis Suppl 69: 19-36.

38. Smith JS, Robinson NJ (2002) Age-specific prevalence of infection with herpes simplex virus types 2 and 1 . J Infect Dis 186: S3-28.

39. Yolken RH, Torrey EF (2006) Infectious agents and gene-environmental interactions in the etiopathogenesis of schizophrenia. Clinical Neuroscience Research 6: 97-109

40. Hinze-Selch D, Becker EW, Stein GM, Berg PA, Mullington J, et al. (1998) Effects of clozapine on in vitro immune parameters: a longitudinal study in clozapine-treated schizophrenic patients. Neuropsychopharmacology 19: 114-22.

41. Grayston JT, Campbell LA, Kuo CC, Mordhorst CH, Saikku P, et al. (1990) A new respiratory tract pathogen:Chlamydia pneumoniae strain TWAR J Infect Dis 161: 618-625.

42. Subtil A, Dautry-Varsat A (2004) Chlamydia: five years AG (after genome). Curr Opin Microbiol 7: 85-92.

43. Aslan M, Kasapcopur O, Yasar H, Polat E, Saribas et al. (2011) Do infections trigger juvenile idiopathic arthritis? Rheu Int 31: 215-220.

44. Sundelöf B, Gnarpe H, Gnarpe J (1993) "An unusual manifestation of Chlamydia pneumoniae infection: meningitis, hepatitis, iritis and atypical erythema nodosum," Scand J Infect Dis 25: 259-261.

45. Balin BJ, Appelt DM (2001) Role of infection in Alzheimer's disease. J Am Osteopath Assoc 101: 1-6.

46. Sriram S, Yao S, Stratton CW, Mitchell WM, Calibresi P, et al. (2002) Comparative study of the presence of Chlamydia pneumoniae in cerebrospinal fluid of patients with clinically definite and monosymptomatic multiple sclerosis Clin Diagn Lab Immunol 9: 1332-1337.

47. Pozniak AL, Lobzin IuV, Mikhailenko AA, Mudritskii VM, Nuralova IV, et al. (2002) Acute chlamydial lesions of the nervous system: etiology, diagnosis clinical aspects. Clin Med (Mosk) 80: 31-34.

48. Reis J, Le Faou A, Levy F, Kapfer MT, Gut JP (1985) Confusional form of Chlamydia psittaci encephalitis. Diagnostic value of microimmunofluorescence. A case. Presse Med 14: 87-89.

49. Brewis C, McFerran DJ (1997) 'Farmer's ear': sudden sensorineural hearing loss due to Chlamydia psittaci infection. J Laryngol Otol 111: 855-857.

50. McAllister CG, van Kammen DP, Rehn TJ, Miller AL, Gurklis J, et al. (1995) Increases in CSF levels of interleukin-2 in schizophrenia:effects of recurrence of psychosis and medication status. Am J Psychiatry 152: 1291-1297.

51. Debattista J, Timms P, Allan J, Allan J (2003) Immunopathogenesis of Chlamydia trachomatis infections in women. Fertil Steril 79: 1273-1287.

52. Ebadi M, Bashir RM, Heidrick ML, Hamada FM, Refaey HE, et al. (1997) 
Citation: Kocazeybek B, Kalayci F (2015) Assessment of Association between Schizophrenia and Chlamydiaceae Using Hill Criteria. J Psychiatry 18: 250 doi: 10.4172/2378-5756.1000250

Neurotrophins and their receptors in nerve injury and repair. Neurochem Int 30: 347

53. Frost DO, Tamminga CA, Medoff DR, Caviness V, Innocenti G, et al. (2004) Neuroplasticity and schizophrenia. Biol Psychiatry 56: 540-3.

54. Shoval G, Weizman A (2005) the possible role of neurotrophins in the pathogenesis and therapy of schizophrenia. Eur Neuropsychopharmacol 15: 319-29.

55. Elkabes S, DiCicco-Bloom EM, Black IB (1996) Brain microglia/macrophages express neurotrophin that selectively regulate microglial proliferation and function. J Neurosci 16: 2508-2521.

56. Miwa T, Furukawa S, Nakajima K, Furukawa Y, Kohsaka S (1997) Lipopolysaccharide enhances synthesis of brain-derived neurotrophic factor in cultured rat microglia J Neurosci Res 50: 1023-1029.

57. Besser M, Wank R (1999) Cutting edge: clonally restricted production of the neurotrophins brain-derived neurotrophic factor and neurotrophin-3 mRNA by human immune cells and Th1/Th2-polarized expression of their receptors. J Immunol 162: 6303-6306.

58. Moalem G, Gdalyahu A, Shani Y, Otten U, Lazarovici P, et al. ( 2000) Production of neurotrophins by activated $T$ cells: implications for neuroprotective autoimmunity. J Autoimmun 15: 331-345.

59. Kalayci $F(2014)$ the role of Chlamydophila pneumoniae in the ethiopatogenesis of schizophrenia: relationship between schizophrenia and brain derived neurotrophic factor (BDNF) and neurotrophin-3 (NT-3). Unpublished Master Thesis. University of Istanbul, Istanbul, Turkey.

60. Fellerhoff B, Wank R (2011) Increased prevalence of Chlamydophila DNA in post-mortem brain frontal cortex from patients with schizophrenia. Schizophr Res 129: 191-195.

61. Park MH, Kwon YJ, Jeong HY, Lee HY, Hwangbo Y, et al. (2012) Association between intracellular infectious agents and schizophrenia. Clin Psychopharmacol Neurosci 10: 117-123.

62. Krause DL, Weidinger E, Matz J, Wildeneuer A, Wagner JK, et al. (2012) Infectious agents are associated with pyschiatric diseases. Ment IIIn 4: e10.

63. Harkinezhad T, Verminnen K, De BM, Rietzschel E, Bekaert S, et al. (2009) Prevalence of Chlamydophila psittaci infections in a human population in contact with domestic and companion birds. J Med Microbiol 58: 1207-12.

64. Hill AB (1965) The Environment and disease: association or causation? Proc R Soc Med. 58: 295-300

65. Murray RM, Lewis SW (1987) is schizophrenia a neurodevelopmental disorder? BMJ (Clin Res Ed) 295: 681-2.

66. Weinberg SM, Jenkins EA, Marazita ML, Maher BS (2007) Minor physical anomalies in schizophrenia: a meta-analysis.Schizophr Res 89: 72-85.

67. Rapoport JL, Addington AM, Frangou S, Psych MR (2005) the neurodevelopmental model of schizophrenia: update 2005. Mol Psychiatry 10: 434-449

68. Khandaker GM, Zimbron J,Lewis G, Jones PB (2012) Prenatal maternal infection, neurodevelopment and adultschizophrenia: a systematic review of population based studies. Psychological Medicine, Available on CJO.

69. Remington JS, McLeod R, Thulliez P (2006) Toxoplasmosis. In: Remington JS, Klein JO, Wilson CB, Baker C, Eds .Infectious diseases of fetus and newborn Infant.6th ed. Philadelphia: Elsevier Saunders 947-1091.

70. Wright P, Murray RM (1993) Schizophrenia: prenatal influenza and autoimmunity. Annals of Medicine 25: 497-502.

71. Cannon M, Jones PB, Murray RM (2002) Obstetriccomplications and schizophrenia : historical andmeta analytic review. American Journal of Psychiatry 159: 1080-1092.

72. Edwards MJ (1968) Congenital malformations in the rat following induced hyperthermia during gestation. Teratology 1: 173-177.

73. Meyer U, Feldon J (2010) Epidemiology-driven neurodevelopmental animal models of schizophrenia. Progress in Neurobiology 90: 285-326.

74. Owen D, Andrews MH, Matthews SG (2005) Maternal adversity, glucocorticoids and programming of neuroendocrine function and behaviour. Neuroscience and Biobehavioural Reviews 29: 209-26.
75. Bradley AJ, Dinan TG (2010) A systematic review of hypothalamic-pituitaryadrenal axis function in schizophrenia: implications for mortality. Journal of Psychopharmacology 24: 91-118.

76. Meyer U, Feldon J (2009) prenatal exposure to infection: aprimary mechanism for abnormal dopaminergic development in schizophrenia. Psychopharmacology (Berlin) 206: 587602.

77. Hurme M, Haanpaa M, Nurmikko T, Wang XY, Virta M, Pessi T, et al. (2003) IL10 gene polymorphism and herpesvirus infections. Journal of Medical Virology 70 (Suppl. 1): S48-S50

78. Bocchio Chiavetto L, Boin F, Zanardini R, Popoli M,Michelato A, et al. (2002) Association between promoter polymorphic haplotypes of interleukin-10 gene and schizophrenia. Biological Psychiatry 51: 480-484.

79. Grayston JT (1992) Infections caused by Chlamydia pneumoniae strain TWAR Clin Infect Dis 15: 757-761.

80. Tuuminen T, Varjo S, Ingman H, Weber T, Oksi J, et al. (2000) Prevalence of Chlamydia pneumoniae and Mycoplasma pneumoniae immunoglobulin $G$ and $\mathrm{A}$ antibodies in a healthy Finnish population as analyzed by quantitative enzyme immunoassays. Clin Diagn Lab Immunol 7: 734-738.

81. Hinze-Selch D, Pollmacher T (2001) In vitro cytokine secretion in individuals with schizophrenia: results, confounding factors, and implications for further research. Brain Behav Immun 15: 282-318.

82. Singh N, Agrawal S, Rastogi AK (1997) Infectious diseases and immunity: special reference to major histocompatibility complex. Emerg Infect Dis 3: 41-9.

83. Miuller N, Schwarz MJ (2007) The immunological basis of glutamatergic disturbance in schizophrenia: towards an integrated view. J Neural Transm Suppl 72: 269-80.

84. Pozniak AL, Lobzin lu V, Mikhailenko AA, Mudritskii VM, Nuralova IV, et al (2002) Makarov VI. Acute chlamydial lesions of the nervous system: etiology, diagnosis, clinical aspects. Klin Med (Mosk) 80: 31-34.

85. Brewis C, McFerran DJ (1997) 'Farmer's ear': sudden sensorineural hearing loss due to Chlamydia psittaci infection. J Laryngol Otol 111: 855-857.

86. Reis J, Le Faou A, Levy F, Kapfer MT, Gut JP (1985) Confusional form of Chlamydia psittaci encephalitis. Diagnostic value of microimmuno fluorescence. A case. Presse Med 14: 87-89.

87. Schlossberg D (2000) Chlamydia psittaci (psittacosis). In: Mandell GL,Bennet JE, Dolin R (eds). Principle and Practice of Infectious Diseases. Churchil Livingston: Philadelphia 2004-2007.

88. Bébéar C, de Barbeyrac B (2009) Genital Chlamydia trachomatis infections Clin Microbiol Infect 15: 4-10.

89. Mowafy MA, Saad NE, El-Mofty HM, ElAnany MG, Mohamed MS (2014) The prevalence of Chlamydia trachomatis among patients with acute conjunctivitis in Kasr Alainy ophthalmology clinic. Pan Afr Med J 17:151.

90. Hammerschlag MR, Roblin PM, Cummings C, Williams TH, Worku M, et al (1987) Comparison of enzyme immunoassay and culture for diagnosis of chlamydial conjunctivitis and respiratory infections in infants. J Clin Microbiol 25: 2306-8.

91. Schmidt BJ, Hermansen MN (2013) Late debut of neonatal pneumonia caused by Chlamydia trachomatis. Uqeskr Laeger 175: 510-1.

92. Odeh M, Oliven A, Rauchfleisch S, Bassan H (1991) Dilated cardiomyopathy associated with Chlamydia trachomatis infection. J Intern Med 229: 289-291.

93. Hawkins DA, Thomas BJ, Munday PE (1983) Meningoencephalitis associated with Chlamydia trachomatis infection. Br Med J (Clin Res Ed) 286: 1146.

94. Levitt D, Danen R, Levitt P (1986) Selective infection of astrocytes by Chlamydia trachomatis in primary mixed euron-glial cell cultures. Infect Immun 54: 913-916.

95. Wank R (2002) Schizophrenia and other mental disorders require long-term adoptive immunotherapy. Med Hypotheses 59: 154-158.

96. Fellerhoff B, Laumbacher B, Wank R (2005) High risk of schizophrenia and other mental disorders associated with chlamydial infections: hypothesis to combine drug treatment and adoptive immunotherapy. Med Hypotheses 65: 243-252.

97. Frykholm BO (2009) on the question of infectious aetiologies for multiple sclerosis, schizophrenia and the chronic fatigue syndrome and their treatment with antibiotics. Med. Hypotheses 72: 736-739. 